\title{
The geometry, topology and structure of amorphous solids
}

\author{
Cheuk-Yu Lee ${ }^{\text {a }}$, Zbigniew H. Stachurski ${ }^{\text {a,* }}$, T. Richard Welberry ${ }^{b}$ \\ ${ }^{a}$ College of Engineering and Computer Science, Australian National University, Canberra, ACT 0200, Australia \\ ${ }^{\mathrm{b}}$ Research School of Chemistry, Australian National University, Canberra, ACT 0200, Australia
}

Received 21 July 2009; received in revised form 21 September 2009; accepted 21 September 2009

Available online 21 October 2009

\begin{abstract}
Clusters of atoms can be divided into three categories depending on their topology. One of the categories provides the basis for development of a model of a perfectly random structure (ideal amorphous solid) using the non-equilateral triangle topology in the coordination shell. Metallic glasses solidify as amorphous solids with random arrangement of atoms. A model of Zr-based metallic glass has been constructed and described in terms of cluster topology, and compared with a recently published dynamic molecular model of the same alloy. It is shown that the pair distribution function for the ideal amorphous model relates to the pair correlation function obtained from the dynamic model. Debye X-ray scattering computations reveal the presence of vacancies and other flaws relative to the ideal amorphous solid. A shift in the peak position can be predicted using the Erhenfest formula. Two atomic displacement mechanisms involving a five-atom sub-cluster are identified as the fundamental means of compositional redistribution between clusters in the alloy.

(C) 2009 Acta Materialia Inc. Published by Elsevier Ltd. All rights reserved.
\end{abstract}

Keywords: Cluster topology; Multiatomic glass; Debye scattering; Amorphousness

\section{Introduction}

The understanding of the atomic-scale structure of solids (from which modern technology and society benefit so greatly) has come about to a large degree because of the development of the methods of geometry and X-ray crystallography. From the first discovery of the diffraction of X-rays by a crystal in 1912 [1] to the present day, when structures of large protein molecules are determined routinely, crystallography has developed from small beginnings to become an enormously successful and powerful tool. Of pivotal importance in this success is the fact that the methods of crystallography are based on the concept of an ideal (perfect) crystal in which unit cells or building blocks of the material are stacked in perfectly repeating rows and columns to form a periodic array of atoms or molecules (of infinite extent). This same ideal is assumed for the very simplest to the most complex of structures.

\footnotetext{
* Corresponding author. Tel.: +6126125 5681; fax: +61261250506. E-mail address: zbigniew.stachurski@anu.edu.au (Z.H. Stachurski).
}

Although real crystals only ever approximate this ideal (some not very closely), it is this ideal structure which is always used as a permanent baseline relative to which real crystalline materials are compared and can be understood.

In 1984 a new class of solid materials were discovered [2] that possessed long-range orientational order but no translational symmetry. These so-called quasicrystals gave sharp diffraction peaks like crystals but had symmetries incompatible with those found for normal crystals. As this field has developed over the last 25 years, a model that has been used extensively to understand the structure of these novel materials is the Penrose tiling model [3]. This structure is envisaged to be made up of two different types of building blocks (tiles), which take the place of the single unit cell of the ideal crystal model. Although real quasicrystals do not conform exactly to the ideal Penrose model, it nevertheless plays a role of prime importance in providing the same kind of baseline relative to which real quasicrystals may be understood.

For the third class of solid materials, namely amorphous or glassy materials, the situation has not been so 
satisfactory and there is still much debate concerning the exact nature of these materials. In a recent article, Sheng et al. [4] say that "the atomic arrangements in amorphous alloys remain mysterious at present". A search of recently published literature on atomic structure of metallic glassy alloys will reveal that the main effort towards solving this problem is directed towards atomistic molecular dynamics simulations. Since glassy materials are non-equilibrium structures, one should anticipate that the results of each simulation, carried out in different laboratories, will be different; no asymptotically unique structure can be achieved. In light of the above, one can conjecture that this is not the right approach to define the ideal baseline model for the structure of amorphous metals. Instead, a geometrical model should be sought. Such a geometrical ideal amorphous solid (IAS) for mono-sized spheres has been described in detail elsewhere [5], and we follow that approach here to describe the ideal amorphous structure of a Zr-based metallic glass. Coincidentally, a molecular dynamics model of the very same $\mathrm{Zr}$-based metallic glass has been just published by Hui et al. [6], and this gives a special opportunity for comparison and analysis of what are at present the best atomistic dynamic model, with the best theoretical baseline model for this material.

\section{Geometric simulations}

\subsection{IAS model of $\left[\mathrm{Zr}_{41.2} \mathrm{Ti}_{13.8} \mathrm{Cu}_{12.5} \mathrm{Ni}_{10} \mathrm{Be}_{22.5}\right]$ metallic glass}

According to the specific IAS rules [5], the construction of random packing of spheres of $m$ types is as follows:

Step 1: Begin by setting up $m$ virtual bins, each bin containing enough of each type of sphere.

Step 2: Place one sphere of any type at the origin $(x=y=z=0)$.
Step 3: Place $k$ spheres (normally $\bar{k}$ ) in contact with the sphere at the centre, forming a cluster of random configuration. It is essential to use the method of division of the sphere's surface into equal areas [7] to avoid bias (see Fig. 1). Pick up spheres from the bins with a frequency in proportion to the required composition.

Step 4: Identify all three-adjacent-sphere sites formed on the surface of the created cluster and sort the sites in ascending order of distance from the origin.

Step 5: Add spheres of $m$ type on identified sites corresponding to the required composition and in order from the closest to the furthermost from the centre. Reject overlapping spheres.

Step 6: Repeat the previous two steps as many times as required; in principle, the additions can be carried out to infinity; in practice, the simulation ends with a cell of finite dimensions.

The outcome of this computational process is a threedimensional geometrical pattern of randomly packed spheres, called a "Round Cell" if it is of finite dimensions or an IAS if it is of infinite extent. The essential information about the Cell is stored in a matrix, $\left[\mathbf{x}_{n}, m\right]$, where the vectors $\mathbf{x}_{n}$ define the positions of all atoms/spheres with respect to the origin and $m$ identifies the corresponding type of the sphere, including its radius. It is a structure of special geometrical and topological properties. As a rule, the centres of any three adjacent spheres form triangles of unequal sides due to (i) some/all spheres not touching and (ii) different sphere radii. There is not a single incidence of four adjacent spheres that are coplanar, in direct contrast to any of the crystallographic Bravais lattices. Consequently, there is no translational symmetry in this structure. The geometrical construction of IAS lends itself readily to description and analysis by Voronoi tessellation and associated Delauny simplexes. This includes structure
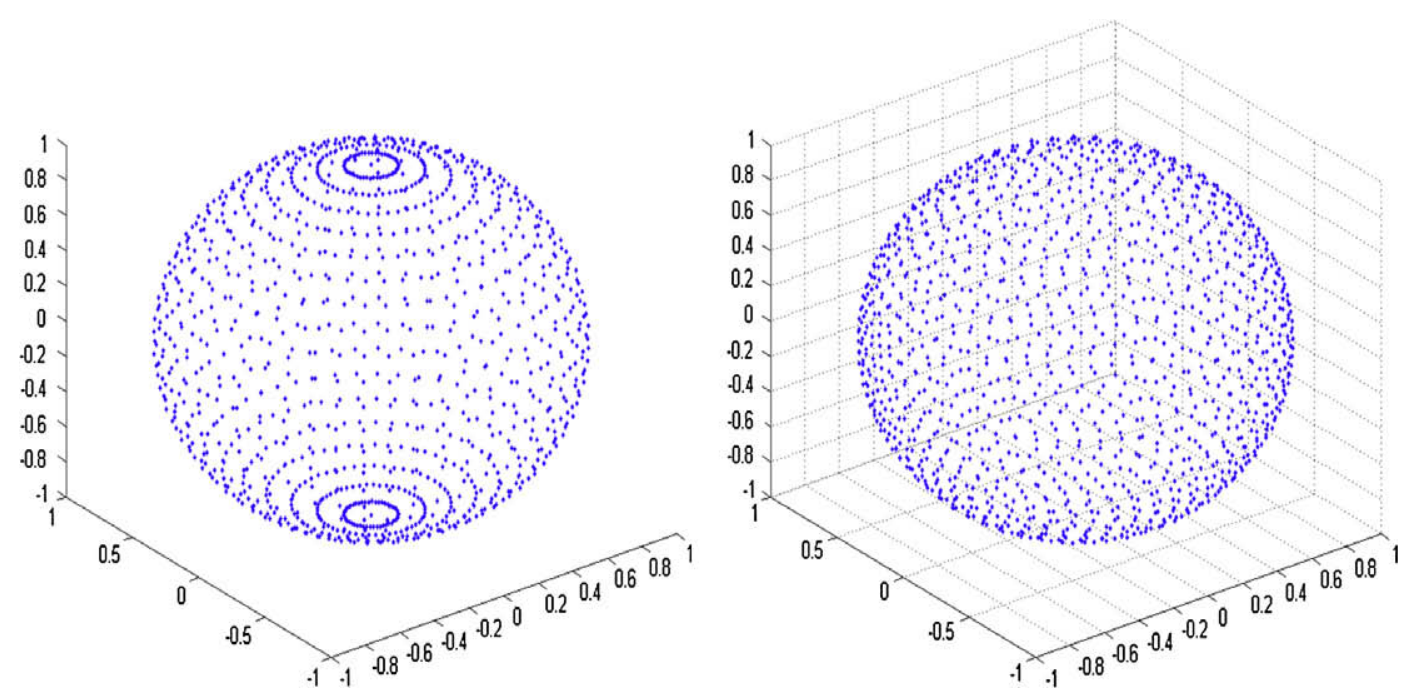

Fig. 1. Density of points on the surface of a sphere: (a) according to the equal area scheme [7] and (b) according to equal probability for the spherical variables: $0 \leqslant \Theta \leqslant \pi, 0 \leqslant \phi \leqslant 2 \pi$. 


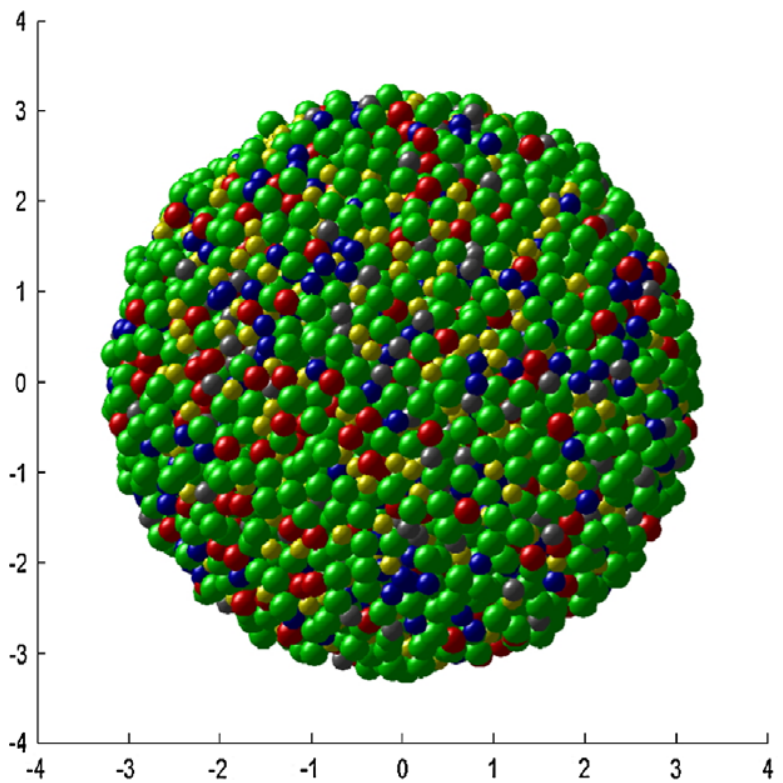

Fig. 2. Round Cell (computer simulated model) for the bulk metallic glass of five elements ( $\mathrm{Zr}$, green; $\mathrm{Ti}$, red; $\mathrm{Cu}$, blue; $\mathrm{Ni}$, grey; and $\mathrm{Be}$, yellow), created by the IAS method. It comprises approximately 7000 spheres randomly packed according to the scheme described herein. (For interpretation of the references to colour in this figure legend, the reader is referred to the web version of this article.)

recognition method developed by Brostow et al. [8]. A large number of tetrahedral Delauny simplexes and a large size of three-type clusters indicates that the solid has a glassy structure. Moreover, an amorphous structure has disordered tetrahedra, as is the case for the Round Cell described above. Other topological properties of the structure will be discussed later.

For the metallic glass $\left[\mathrm{Zr}_{41.2} \mathrm{Ti}_{13.8} \mathrm{Cu}_{12.5} \mathrm{Ni}_{10} \mathrm{Be}_{22.5}\right]$, an ideal amorphous atomic arrangement can be represented by the IAS random packing of five different size spheres. The spheres have radii chosen to correspond to the atomic radii of the elements $(\mathrm{Zr} 0.159, \mathrm{Ti} 0.145, \mathrm{Cu} 0.128, \mathrm{Ni}$ 0.125 , Be 0.114 , in $\mathrm{nm}$ ). We have simulated the corresponding Round Cell model, shown here in Fig. 2, and measured the geometrical and topological properties of this arrangement. The atomic packing density of this Round Cell is $0.625( \pm 0.003)$ for a total of approximately 7000 spheres.

The condition of rigidity/solidity is implicit in the concept of an ideal amorphous solid that has no flaws (the same is true for an ideal crystalline solid). The rigidity of every sphere with $k$ contacts is achieved when no more than $(k-1)$ contacts occur on one hemisphere. Then the sphere is immovable (locked-in) in its cage formed by the surrounding spheres [9]. A less axiomatic measure of solidity, or solid-like character of the structure, is provided by a criterion based on the Delauny simplex circumradii requiring that solid-like percolative clusters exist in all glass phase models, such as the IAS considered here [10]. This is indeed the case for the IAS since it does not contain any loose spheres.

The coordination numbers, based on touching neighbours, will be range-bound to $k_{\text {min }} \leqslant k \leqslant k_{\text {max }}$, where $k_{\min } \geqslant 4$, and the value of $k_{\max }$ will depend on the composition of the IAS and the specific cluster selected. To a first approximation one can use the following values for $k_{\max }$ as shown in Table 1. These are listed as a function of the ratio $r_{o} / r_{i}$, relying on the data for $l(r)$ from Table 3 from Clare and Kepert [11].

Thus, if there were a cluster with $\mathrm{Zr}$ as the inner sphere and $\mathrm{Be}$ as all the outer spheres, then theoretically it could have $18 \mathrm{Be}$ atoms in a random (tight) arrangement touching the $\mathrm{Zr}$ inner atom. However, the probability of such a cluster occurring is negligible, because of the of Fürth combinatorics calculations carried out previously [5,12], and because a cluster of this composition is very unlikely in a system comprising five different types of atom. The majority of clusters will have a mix of spheres of different types as shown by the results of Hui et al. (their Fig. 8b).

The measured distribution of coordination numbers for the IAS, based on touching contacts between the spheres, and on Voronoï tessellations, is shown in Fig. 3. We can see that the coordination shell obtained by the Voronoï tessellation method includes on average seven spheres more than the primary, direct contact shell, which is expected [8]. When compared to the result of Hui et al. shown in their Fig. 8a, we note that relaxations towards equalization of interatomic distances closes off some gaps in the clusters, lowers the number of Voronoï faces and consequently lowers the coordination number relative to the rigid IAS structure.

For a system of touching spheres in random arrangement, the contact probability between species $i$ and $j$ can be predicted by the probabilistic formula:

$P_{i j}=\delta c_{i} c_{j}$

where $\delta=2$ for $i=j$ or $\delta=1$ for $i \neq j$, and $c_{i}$ and $c_{j}$ are the concentrations of species $i$ and $j$, respectively. This is a straightforward calculation for a given composition. Theoretically, the same information should be obtained

Table 1

Maximum possible number of outer spheres, $k_{\max }$, that can be in contact with an inner sphere in a cluster (the radius of the outer spheres is $r_{o}$, and the radius of the inner sphere is $r_{i}$ ).

\begin{tabular}{llllllllllll}
\hline$k_{\max }$ & 8 & 9 & 10 & 11 & 12 & 13 & 14 & 15 & 16 & 17 \\
\hline$l(r)[11]$ & 1.22 & 1.15 & 1.09 & 1.05 & 1.05 & 0.96 & 0.93 & 0.90 & 0.88 & 0.86 & 0.84 \\
$r_{o} / r_{i}$ & 1.56 & 1.35 & 1.20 & 1.10 & 1.0 & 0.92 & 0.87 & 0.82 & 0.79 & 0.76 & 0.72 \\
\hline
\end{tabular}




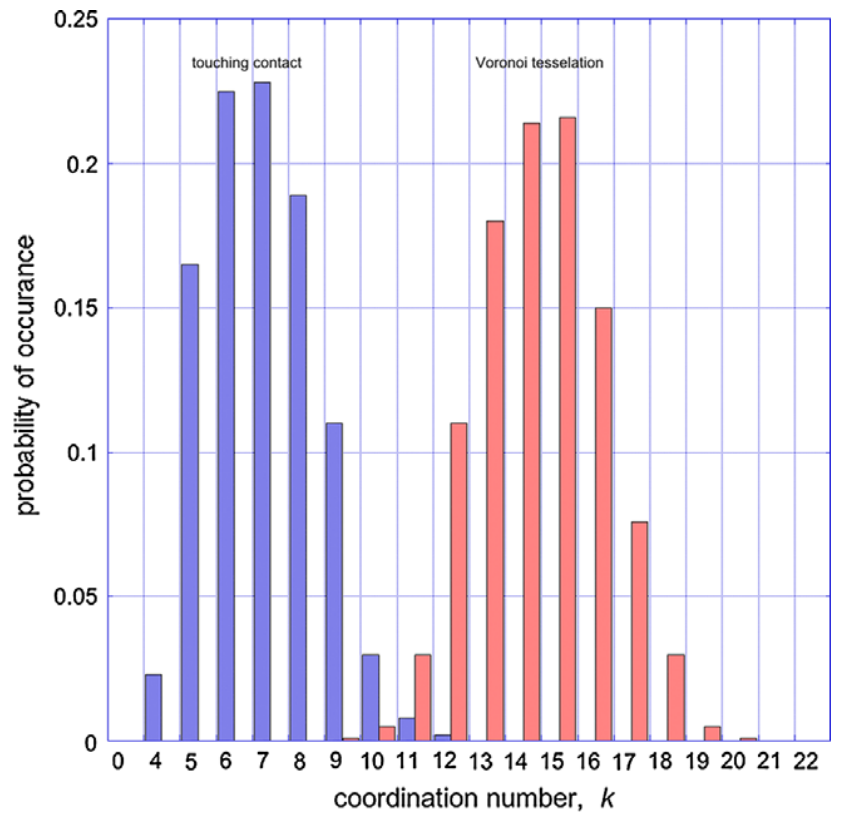

Fig. 3. Measured distribution of coordination numbers in the $\mathrm{Zr}$-based Round Cell: by direct contact (blue) and by Vorono tessellation (red) as indicated in the diagram. (For interpretation of the references to colour in this figure legend, the reader is referred to the web version of this article.)

from calculations of the pair distribution function by the formula:

$g_{i j}(r)=\frac{V}{N_{i} N_{j}} \sum_{i=1}^{N_{i}} \frac{n_{i j}(r)}{4 \pi^{2} \Delta r}$

The result for $g_{i j}$ with a high resolution of $\Delta r=0.001 \mathrm{~nm}$ is shown in Fig. 4; the comparison of $P_{i j}$ calculated from Eq. (1) vs. $g_{i j}$ derived from Eq. (2) is shown in Fig. 5. A

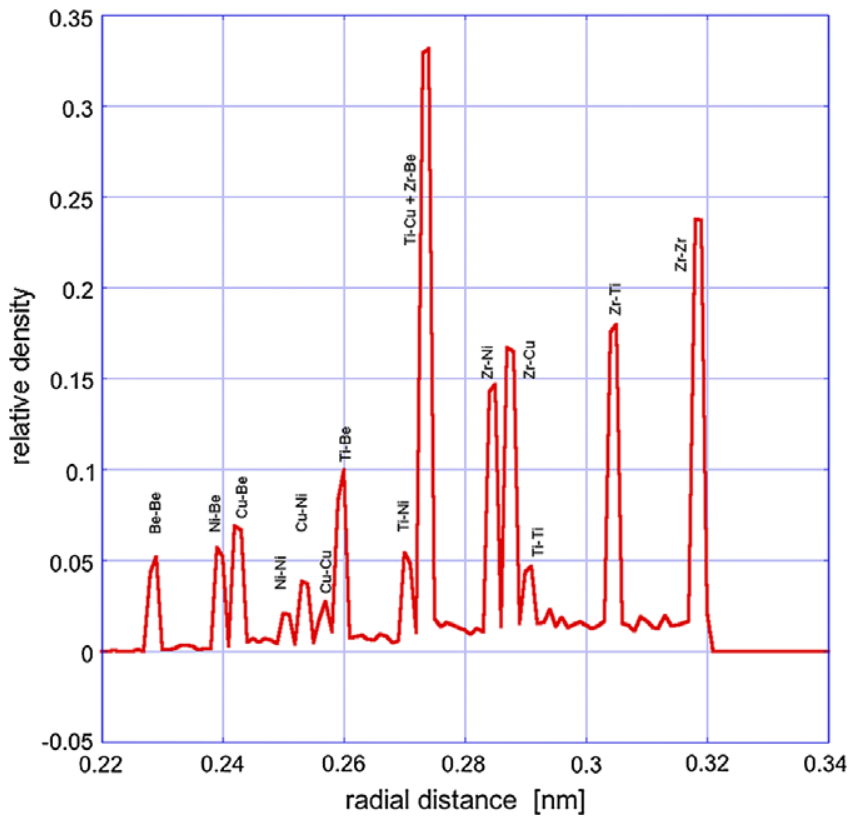

Fig. 4. Radial pair distribution function for the Round Cell of Fig. 2, calculated according to Eq. (2) with radial increment of $0.001 \mathrm{~nm}$. Pair peaks appear individually due to the high resolution.

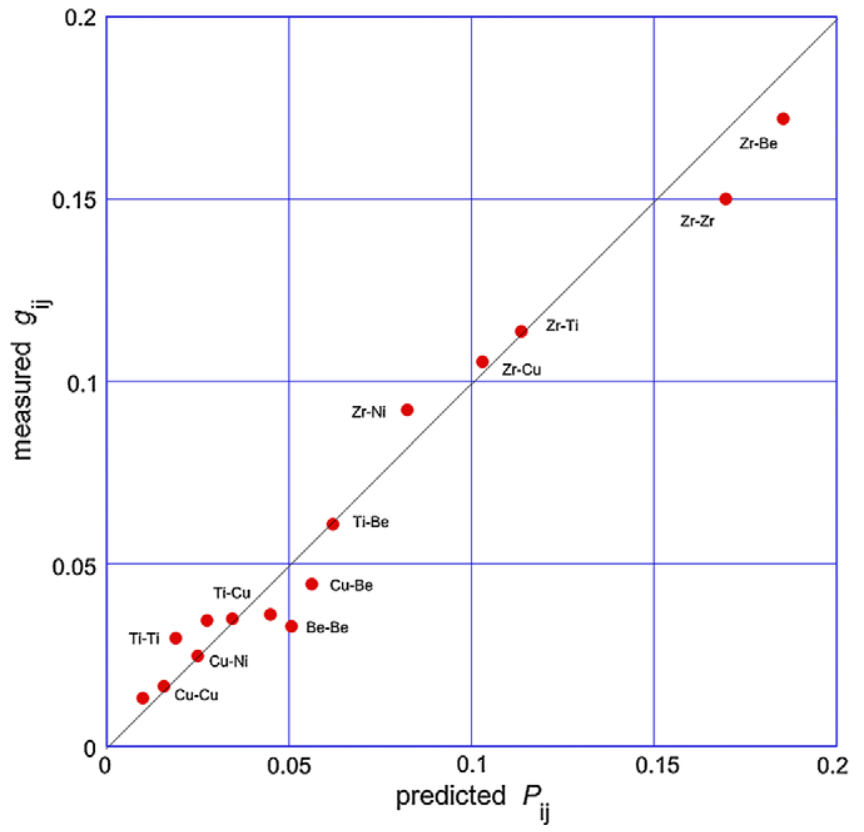

Fig. 5. Probability of contacts between the elements predicted by Eq. (1) compared with the relative density of pairs measured in Fig. 4. One-to-one correspondence is expected.

one-to-one correlation should be expected. Some deviations from the linear relationship are thought to be due to the relatively small size of the simulated Round Cell and to the fact that the outermost layer was not counted in.

To illustrate the difficulties of interpretation of pair distribution functions we have calculated it again for the Round Cell using Eq. (2) with a resolution of $\Delta r=$ $0.026 \mathrm{~nm}$, with the result shown in Fig. 6. The lower

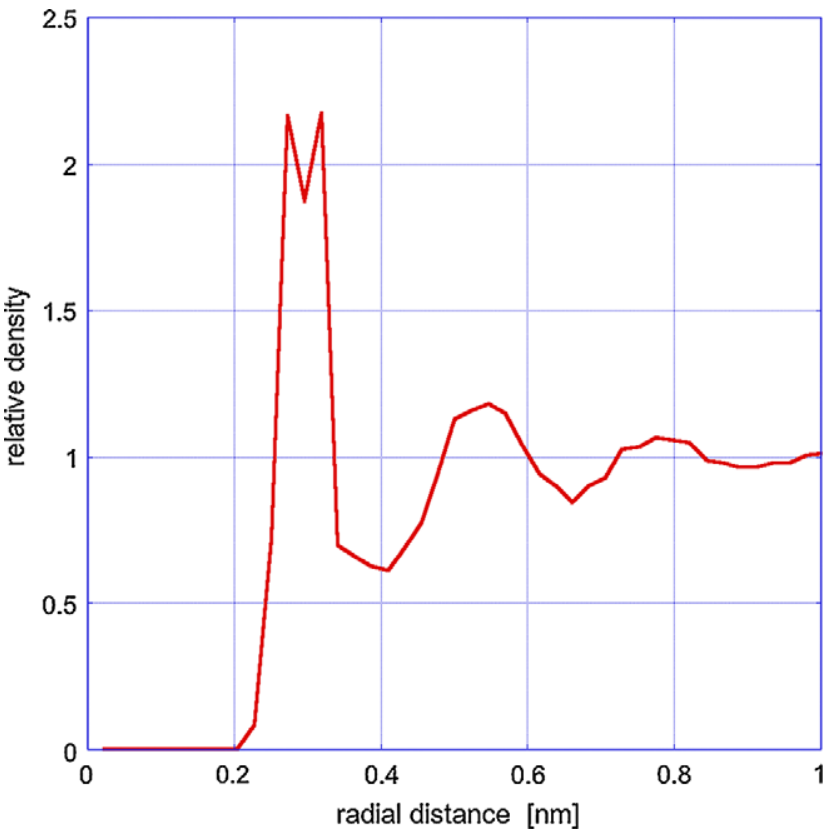

Fig. 6. Radial pair distribution function for the Round Cell of Fig. 2, calculated with a lower resolution (radial increment of $0.026 \mathrm{~nm}$ ). 

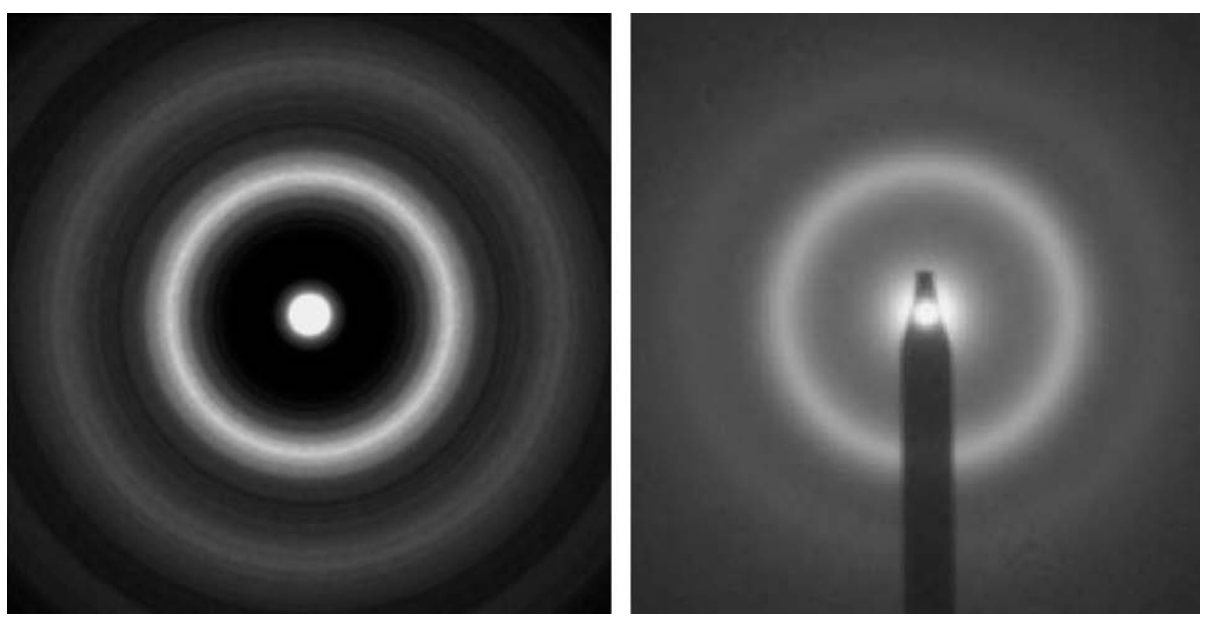

Fig. 7. X-ray scattering patterns from amorphous structures: (left) calculated using Eq. (3) for the Round Cell of the Zr-based glass and (right) experimental results from a $\mathrm{Zr}$-based metallic glass [14].

resolution has the effect of "smearing" of the peaks, thus concealing detailed information. The individual peaks appearing in Fig. 4 have now merged into one broad peak (albeit with a split) between 0.22 and $0.32 \mathrm{~nm}$, which includes contributions from all atomic pairs. The first peak of the split (at approx. $0.27 \mathrm{~nm}$ ) corresponds to $\mathrm{Zr}-\mathrm{Be}$ and $\mathrm{Ti}-\mathrm{Cu}$ pairs, and the second (at approx. $0.32 \mathrm{~nm}$ ) to the other $\mathrm{Zr}-\mathrm{X}$ pairs. The remaining, much less abundant, pairs with distances between 0.22 and $0.32 \mathrm{~nm}$ also contribute to the body of the peak. The second peak in the pair distribution function (at approximately $0.55 \mathrm{~nm}$ ) has no split, which indicates the absence of any regular face-centred cubic or hexagonal close-packed (hcp) cluster arrangements in the IAS. We have provided a proof that the IAS has no short-, medium- or long-range order in a previous publication [5].

\subsection{X-ray scattering from $\mathrm{Zr}$-based IAS}

Given the wavelength of radiation, $\lambda$, the positions of all atoms $\left[\mathbf{x}_{n}\right]$, and the atomic scattering factors, $f_{n}$, then the scattered intensity, $I$, in a given direction of observation, $\mathbf{S}$, can be calculated using the Debye equation:

$$
I(\mathbf{S})=\sum_{n} \sum_{n \neq n^{\prime}} f_{n} f_{n^{\prime}} \exp \left[2 \pi\left(\mathbf{s}-\mathbf{s}_{\mathbf{0}}\right) \cdot\left(\mathbf{x}_{\mathbf{n}}-\mathbf{x}_{\mathbf{0}}\right)\right]
$$

We have computed X-ray scattering from the Round Cell of Fig. 2 using the software DISCUS [13]. The input into the program includes the matrix $\left[\mathbf{x}_{n}, m\right]$, and the corresponding atomic scattering factors are selected automatically. The scattering computed from a single realization of the Round Cell comprising only 7000 atoms is very noisy. In order to improve the statistics, and hence the smoothness of the pattern, calculations were carried out on a much larger system comprising over 500 copies of the single Round Cell realization, each in a different random orientation. Circular averaging has been used to produce further smoothing. The resulting scattering pattern is shown in Fig. 7a. The characteristic rings seen in the figure concur with the variation of the $\sin \left(k r\left(x_{n}\right)\right) / k r\left(x_{n}\right)$ function, convoluted with appropriate atomic scattering factors, as predicted by the Debye scattering equation. It is also similar to an experimentally obtained scattering pattern for this metallic glass shown in Fig. 7b [14].

Three characteristics in the scattering patterns can be related to the atomic arrangements and imperfections in the amorphous solids:

1. The intensity of the first scattering peak.

2. The position of the first scattering peak.

3 . The background intensity related to the concentration of vacancies.

To investigate these effects, we have simulated vacancies in the IAS model by: (i) removing at random $10 \%$ of atoms, thus creating $10 \%$ of vacancies but retaining the same overall composition and the same average pair distance $r\left(x_{n}\right)$; and (ii) removing all $\mathrm{Cu}$ atoms, thus creating $12.5 \%$ vacancies, consequently also changing the composition of the alloy and increasing the average pair contact distance. The results are shown in Fig. 8a and b. For the introduced content of vacancies, the intensity of the first peak has decreased correspondingly, in agreement with the Debye equation [15] and corroborating the results of Makinson et al. [16]. At the same time, the background intensity has increased with the content of vacancies as predicted by the Laue formula [16], also corroborating the results of Makinson et al.

Removing the $\mathrm{Cu}$ atoms lowers the intensity of the peak, but also causes a shift in the position of the peak (Fig. 8b) in accordance with the Ehrenfest formula proposed by Rachek [17], $2 L_{p} \sin (\theta)=E \lambda$, where $E=1.23$ is the socalled Ehrenfest number and $L_{p}$ is the average distance between the scattering pairs. Using the atomic radii for the metallic alloy, the calculated number-weighted average for the IAS without $\mathrm{Cu}$ atoms increases from an initial 

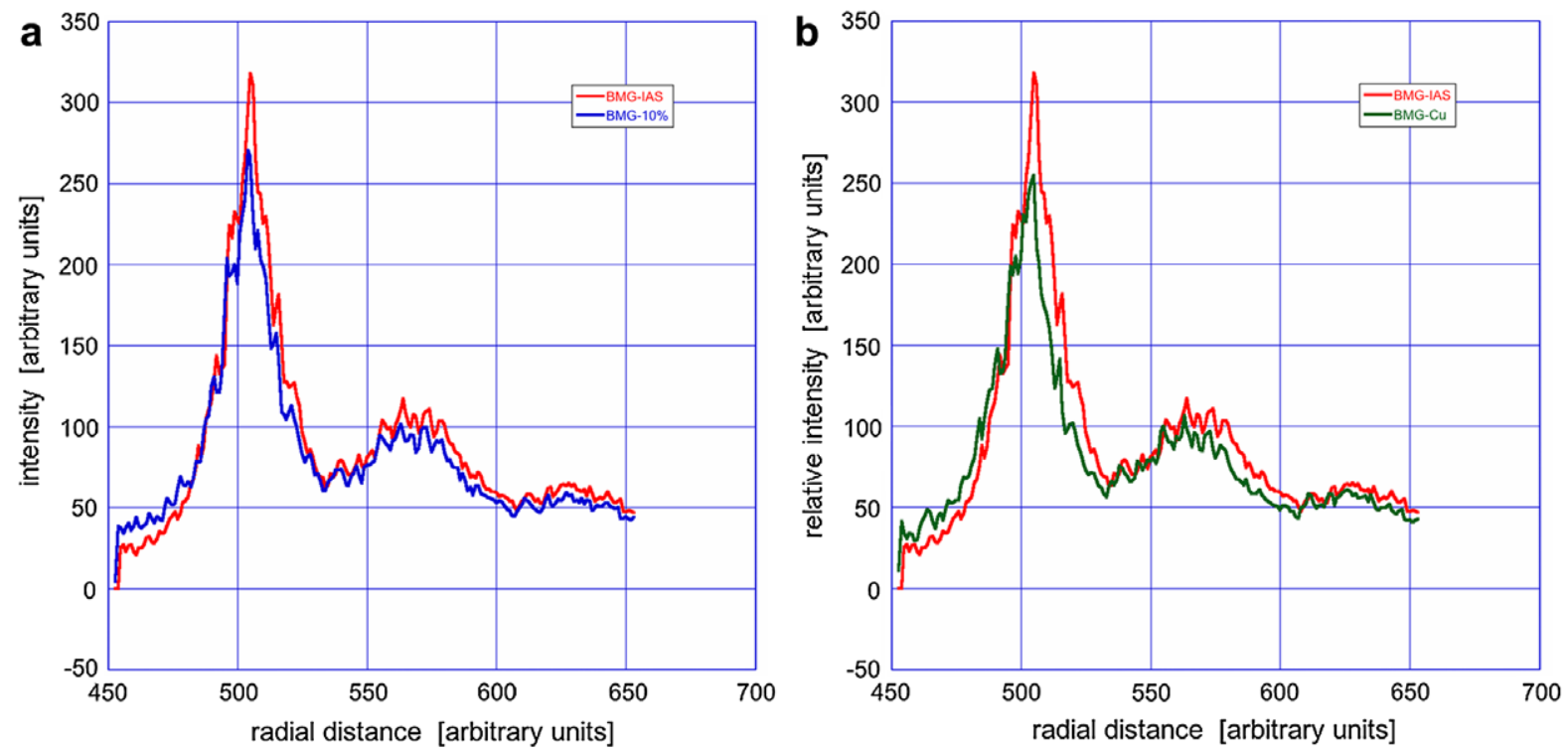

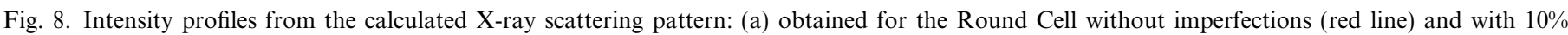

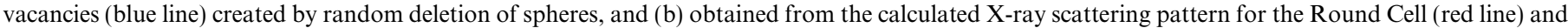

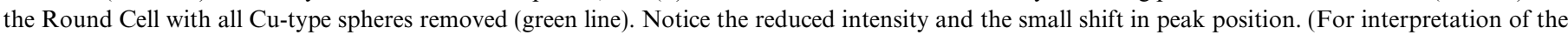
references to colour in this figure legend, the reader is referred to the web version of this article.)

Table 2

Parameters for molecular dynamic simulation based on Lennard-Jones potential.

\begin{tabular}{llll}
\hline $\begin{array}{l}\text { Temperature of } \\
\text { simulation }\left({ }^{\circ} \mathrm{C}\right)\end{array}$ & $\begin{array}{l}\text { Time of } \\
\text { simulation }(\mathrm{s})\end{array}$ & $\begin{array}{l}\text { Number of } \\
\text { steps }\end{array}$ & $\begin{array}{l}L J \\
\text { exponents }\end{array}$ \\
\hline 300 & $10^{-12}$ & 1000 & $24: 6$ \\
\hline
\end{tabular}

value of $0.1412-0.1431 \mathrm{~nm}$. According to the Ehrenfest formula, this should reduce the scattering angle by approximately $1.4^{\circ}$, quite close to the observed shift in Fig. $8 \mathrm{~b}$.

\subsection{Dynamic five-atom sub-clusters}

The Round Cell shown in Fig. 2 was subjected to molecular dynamics simulation, with the parameters shown in Table 2. Irreversible atomic rearrangements were observed and traced. The most consistently striking observations were rearrangements which always involved five-atom sub-clusters and two mechanisms: (i) absorption of an extra atom into a cluster from a neighbouring cluster; or (ii) ejection of an atom into an adjacent cluster. These rearrangements, driven by generalized thermodynamic forces, lead to configurational and compositional changes in the individual clusters.

A five-atom sub-cluster is shown schematically in Fig. 9. It is a segment of the main cluster, including the inner sphere (A) with three adjacent spheres (B-D) from the coordination shell, plus one other sphere (E) touching the three spheres. Atom E actually belongs to an adjacent cluster. In the diagram the spheres are coloured differently but the radii are the same for the sake of simplicity, without reducing generality. The separation between $\mathrm{A}$ and $\mathrm{E}$, indicated as $h$ in the diagram, is variable and depends on the coordination number $k$ of the main cluster, on the spacings between atoms $\mathrm{C}-\mathrm{E}$, which are irregular as a rule, and on their diameters.

Within the elastic matrix of the surrounding atoms, the five-atom sub-cluster will change its shape by the action of interatomic forces, assisted by density differences between adjacent main clusters. When the "hard sphere" regime is relaxed by introduction of central atomic force-fields, the configuration of the five-atom sub-clusters becomes unstable except at three positions. The most stable arrangement occurs when atoms $\mathrm{A}$ and $\mathrm{E}$ separate to a distance $h=4 r \sqrt{2 / 3}$. In this position, atoms B-D touch each other $(d=2 r)$, and the arrangement of the five-atom cluster corresponds to hexagonal close packing. The second stable configuration occurs when atoms $\mathrm{A}$ and $\mathrm{E}$ approach and touch $(h=2 r)$, separating atoms B-D as far as possible to a distance of approximately $d=2 r \sqrt{3}$. Since atoms B$\mathrm{D}$ are in contact with atoms $\mathrm{A}$ and $\mathrm{E}$ at all times, a metastable configuration occurs at some intermediate value of $h$. A calculation for the relative balance of the five-atom cluster, based on Lennard-Jones interactions, is shown in Fig. 10. Rearrangements caused by thermal vibrations will have an immediate effect on changing the initial IAS atomic packing arrangement. It is conjectured that the instability of the five-atom cluster is the main mechanism for morphological changes of the atomic clusters in the $\mathrm{Zr}$-based bulk metallic glass, in the $\mathrm{Nb}_{x} \mathrm{P}_{y}$-type metallic glasses considered by Sheng et al. [4] or in any other system allowing a range of coordinations. Furthermore, it can be envisaged that the $\alpha$ - and $\beta$-mechanisms may be the 

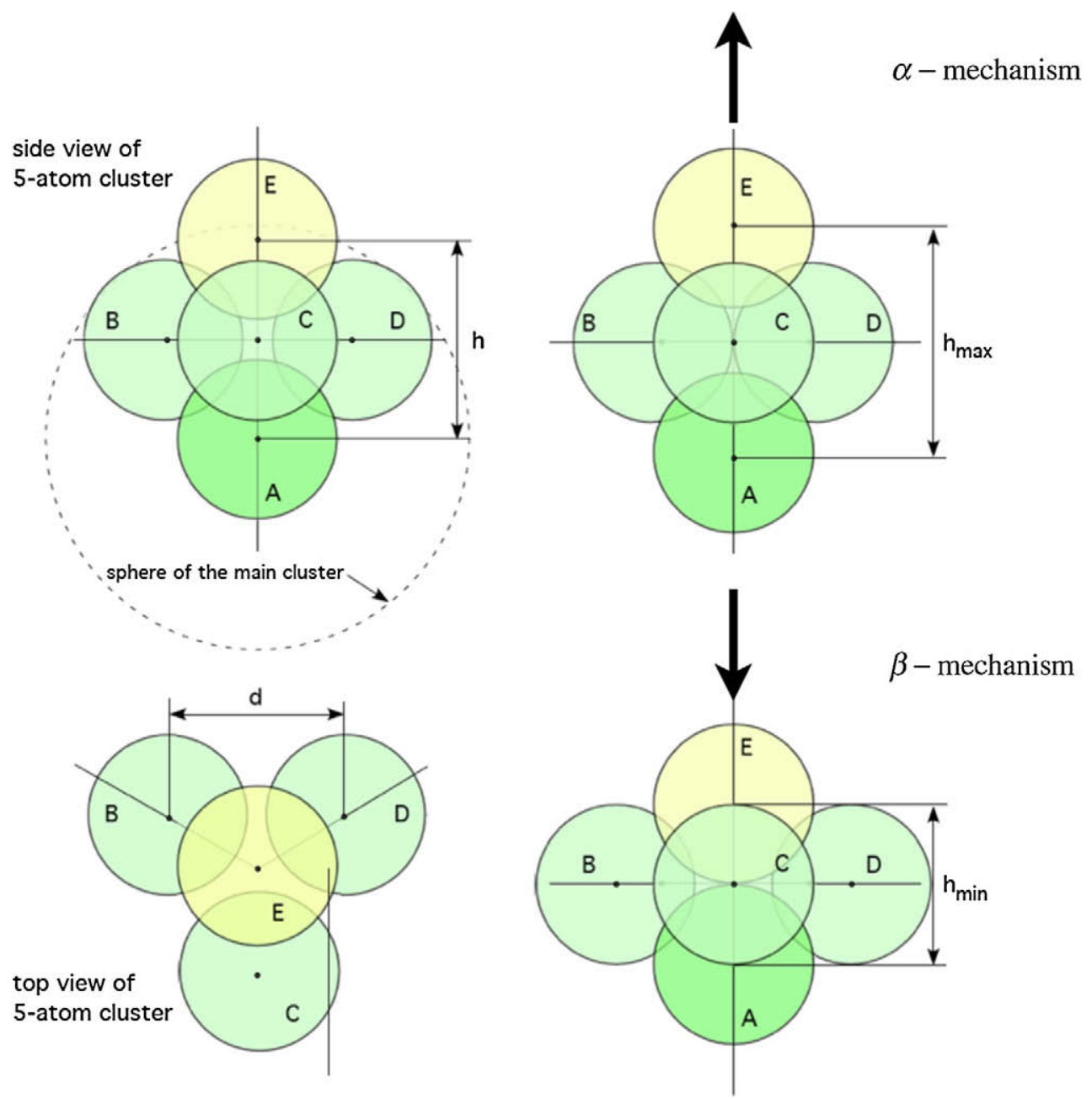

Fig. 9. Schematic diagram of a general five-atom sub-cluster, shown from the side and top views, and also shown in the special $\alpha$ and $\beta$ positions. The spheres are drawn with the same diameters; in multiatomic alloy these will be of different diameters. As a rule, atoms B-D should form a triangle of unequal sides.

elementary atomic motions underlying the main deformation mechanisms, such as local atomic jump and shear transformation zone described in Ref. [18].

\section{Discussion}

\subsection{Cluster topology}

Models of amorphous materials can be divided into two categories: (i) materials with disordered atomic arrangement but retaining some elementary crystallographic features; and (ii) materials with statistically random atomic arrangements and no Bravais lattice. Both approaches use clusters of spheres as the basic building blocks of the structure. A clear distinction can be made between the two approaches if the topology of the clusters is taken into account. Frank and Kasper [19] considered the geometry and topology of clusters of atoms (in preference to crystallographic unit cell) in order to describe the complex atomic arrangements in crystal structures of transition metal alloys. They based their considerations on groupings of atoms comprising an inner (central) atom and a set of closest neighbouring atoms which form the coordination shell. They chose clusters with coordination shells that included triangular faces only. In classifying the clusters they distinguished three cases, depending on the shape of the triangles formed by three adjacent atoms (all atoms of the same size):

Case 1. Coordination shell atoms make equilateral triangles with the centre (inner atom).

Case 2. Triangles in the coordination shell are equilateral; shell atoms make isosceles triangles with the centre.

Case 3. No requirements on the shape of triangles within the shell.

The addition of atoms onto three-adjacent-sphere sites on the clusters of the first case leads directly to the hexagonal close packing of spheres. The second case was of special relevance to the complex crystal structures of transition alloys. Frank and Kasper showed the existence of four spe- 


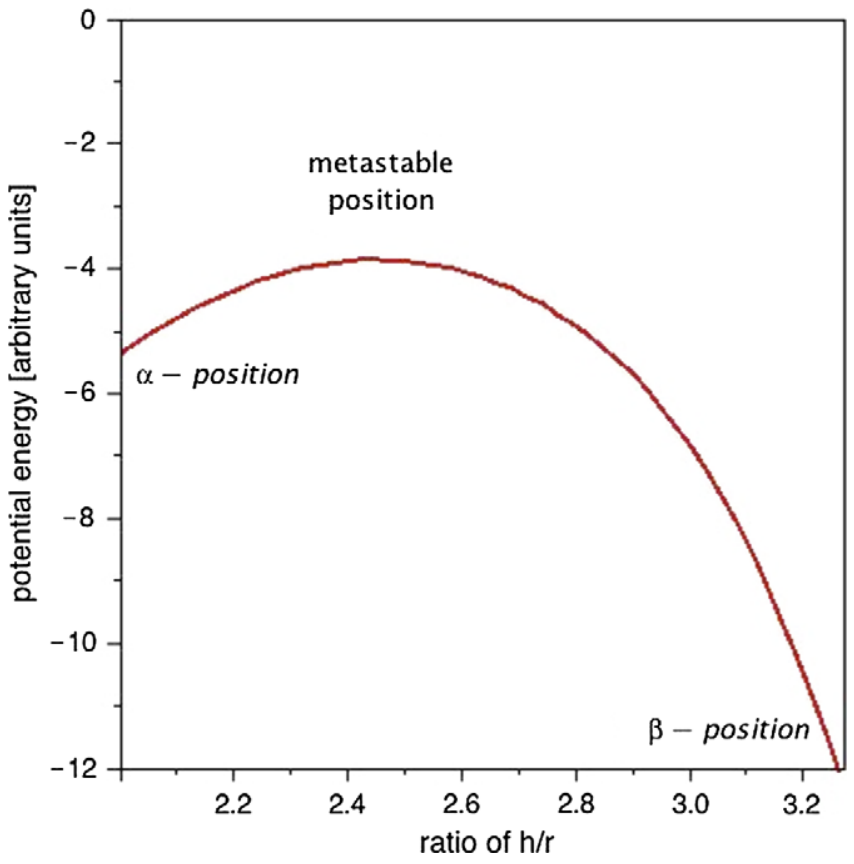

Fig. 10. Variation in potential energy of a five-atom sub-cluster in relation to the atomic separations indicated in Fig. 9.

cial coordination clusters $-\mathrm{CN} 12, \mathrm{CN} 14, \mathrm{CN} 15$ and CN16 - which became known as the Frank-Kasper (FK) polyhedra. The outer shell polyhedra display elements of rotational symmetry imposed by the geometry implied in condition 2 above. The addition of atoms onto three-adjacent-sphere sites onto these clusters creates quasicrystals. Incidentally, the range of FK polyhedra was further extended by Borodin [20], and generalized by Alvarez [21] when deriving principles for construction of concentric shells of fullerene polyhedra.

In metallic glasses the inevitable variance of coordination numbers from cluster to cluster has evoked the idea that atomic arrangements in these alloys could be described in terms of random packing of regular FK polyhedra $[4,6,22]$. Such a structure would account for the seemingly disordered nature of these materials. However, on closer inspection problems with this view become evident. First, the FK polyhedra exhibit rotational symmetry, and, if packed without distortion, relatively sharp X-ray or electron diffraction spots should occur (instead of diffuse scattering rings). This is, in fact, observed when annealing of metallic glass leads to the onset of nanocrystallization [23]. Second, if atomic relaxations are allowed to occur so as to fit the polyhedra contiguously, then they are no longer FK polyhedra satisfying the requirements of case 2 above, and furthermore, the connecting atomic structures will contain polyhedra other than those based on tetrahedral groupings, with quadrangles besides triangles. Strictly speaking, the FK polyhedra are not an appropriate choice to describe amorphous atomic arrangements because the atoms in the coordination shells form equilateral triangles.

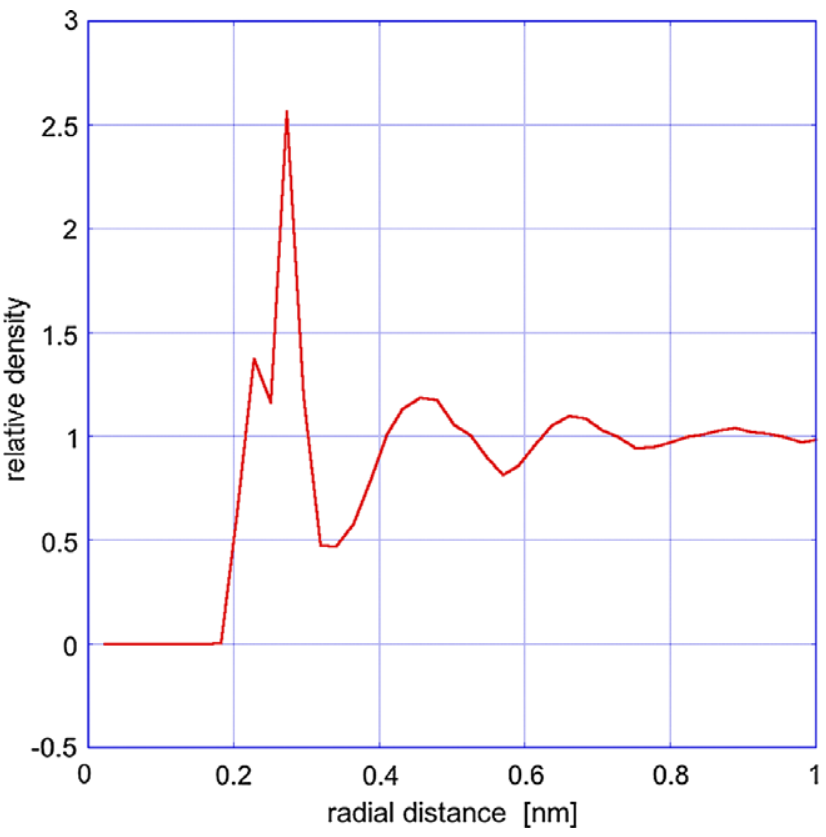

Fig. 11. Pair correlation function for the Zr-based IAS model after being subjected to a molecular dynamics run.

The third case includes clusters with random arrangement with non-equilateral triangles in their coordination shell, according to the third case above. The addition of atoms onto three-adjacent-sphere sites of this cluster can, theoretically, be carried out without limit. We regard this case as the basis for the development of a theoretical model of an ideal amorphous solid. Such an ideal amorphous solid for mono-sized spheres has been described in detail elsewhere [5]. In an IAS of Class I the cluster's polygonal faces have triangular shapes exclusively; any triangle formed by three adjacent spheres in the coordination shell is a non-equilateral triangle, in accordance with case 3 above. Indeed, the first cluster in the construction of the IAS (step 3) is an FK cluster with non-equilateral triangular topology constructed by random placement of the coordination shell spheres. The IAS model of a rigid solid requires the spheres (representing atoms) to be impenetrable and in contact with each other. This is a stricter definition of coordination number than that used by Frank and Kasper and others, which makes the topological arguments well defined.

Hui et al. [6] make the point that different interpretations of the atomic configurations can be elicited from molecular dynamics and ab initio models if no theoretical model is established, and that there is a pressing need for new methodology in modelling the structures of multicomponent BMGs. We believe that the IAS model provides such an unambiguous theoretical baseline reference, as shown already to some extent by the considerations above. We have repeated calculations of the pair distribution function (called more correctly pair correlation function) after subjecting the IAS model to molecular dynamics simulation. The result is shown in Fig. 11. It is very similar to 

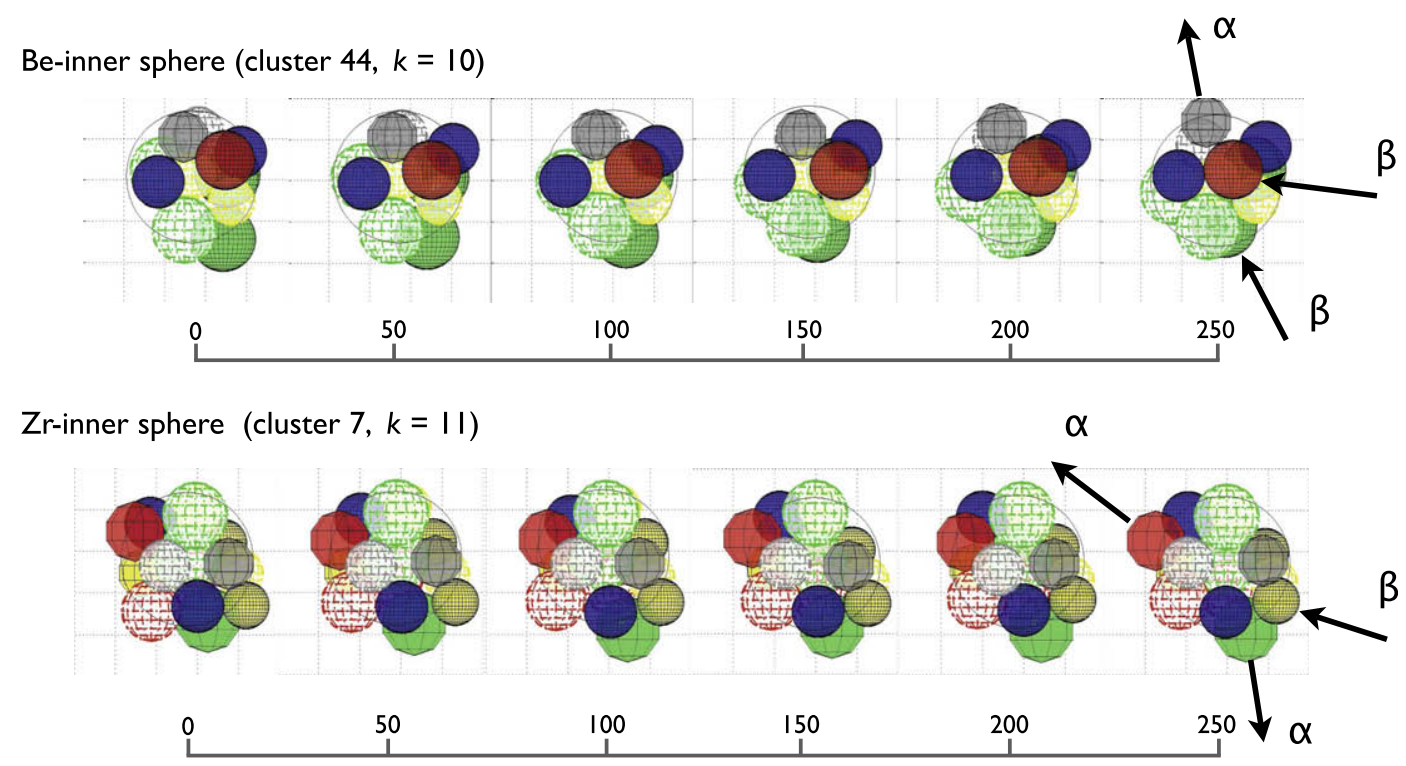

Fig. 12. Two examples of different clusters undergoing configurational changes during molecular dynamics simulation. The $\alpha$ - and $\beta$-mechanisms are clearly identified in these examples. The scale underneath indicates the number of steps in the simulation.

the PCF at $300 \mathrm{~K}$ obtained by Hui et al. and shown in their Fig. 4a. The accuracy of their work corroborates the result and confirms the validity of the IAS model.

\subsection{Atomic rearrangements}

As envisaged, the thermodynamic potential of the IAS structure is higher than that of the more relaxed, dynamic structure based on the same atoms (changes in entropy associated with these rearrangements will only become measurable when significant ordering takes place.) When the Round Cell is subjected to a molecular dynamics (MD) process it is expected that the atomic structure will change and its thermodynamic potential will decrease. Each of the five-atom sub-clusters will be driven towards one of the two stable positions: (i) the less favourable position, which we denote as $\alpha$; and (ii) the more stable position, denoted as $\beta$. In general, clusters with low coordination numbers $(k \leqslant \bar{k})$ will have five-atom sub-clusters which are closer to the $\alpha$ position shown in Fig. 10 at $h /$ $r=2$. Then atom $\mathrm{E}$ will gradually approach atom $\mathrm{A}$ to an equilibrium (touching) separation, and therefore become part of the main cluster centred on atom A. By this process, the coordination number of the main cluster increases and its configuration changes. This effect was also noted by Hui et al. [6] in their Fig. 11 and by Liu et al. [24], who said that "adjustment of the relative atomic sites occurs without any long-distance diffusion". It should occur most vigorously for clusters of lowest coordination number, surrounded by clusters of high coordination numbers (high density). It is presumed that this occurs as a cooperative movement with the surrounding atoms. For further reference, we denote this process as the $\alpha$-mechanism.

Concurrently, other five-atom sub-clusters contained in main clusters with coordination numbers $k \geqslant \bar{k}$ will diffuse towards the $\beta$ position at $h / r=4 \sqrt{2 / 3}(h \approx 3.27)$. This will have a pronounced effect on the configuration of the main cluster, as well as creating a potential hcp embryo that may lead to nanocrystallization by enthalpic attraction of adjacent atoms. As atoms A and E move apart, atoms B-D are drawn together, thus creating gaps between the outer atoms of the main cluster, opening space for diffusion of other atoms towards the inner atom and towards the embryo. This process of rearrangement will be denoted as the $\beta$-mechanism. Examples of the $\alpha$ - and $\beta$-mechanisms are shown in Fig. 12a and b for selected clusters from computer simulations carried out on the bulk metallic glass. The sequence shows the atomic configuration of the cluster in IAS packing, followed by MD at 50, 100, 150, 200 and 250 steps. Such structural changes can be described more precisely in terms of the metamorphosis of Voronoi polyhedra, as given elsewhere [25].

Strong evidence for the random distribution of atoms in the Zr-based alloy was published by Martin et al. [23], who presented elemental maps of all alloying elements in relatively large volume of $5 \times 10^{3} \mathrm{~nm}^{3}$, showing clearly that the elements are spread evenly throughout that volume. The appearance of the so-called $i$-phase in the BMG at an early stage of annealing was also confirmed by the same authors. The sharp diffraction spots with fivefold symmetry from the $i$-phase require the formation of regular icosahedral clusters. This means that, topologically, the clusters must have coordination shell atoms of the same type, and they must be equally spaced to form equilateral triangles (CN12). Such a cluster can act as a nucleus (seed) for the growth on a nanoparticle with fivefold symmetry. Measurements carried out on the nanoparticles indicate volumes containing approximately hundreds of atoms. Martin et al. [23] suggest, on the basis of composition depth profiles, that the $i$-phase 
in this alloy is formed with $\mathrm{Ti}$ as the outer atoms and $\mathrm{Be}$ as the inner atoms. Considering the complexity of the $i$ phase structure and the large number of atoms involved in the quasicrystalline arrangement, it is considered unlikely that the composition of the $i$-phase nanoparticles is made of the two types of atom only. The work of Hui et al. confirms that the extended clusters incorporate more than two atomic species (see their Fig. 10). It is conjectured that the new technique of fluctuation electron microscopy may be a suitable technique to map the compositional variations at the small scale.

\subsection{X-ray scattering and imperfections}

Real solids contain imperfections in their atomic arrangements, whether they are departures from an ideal crystalline or pseudo-crystalline arrangement, or departures from an ideal amorphous arrangement. Thus, perfection is used here in a broader sense, referring to any structure that obeys given rules without exception, whether it applies to Bravais rules for construction of single crystal or IAS rules for construction of a perfectly random atomic arrangement. In this broader sense, a gas in which the particle velocities obey the Maxwell distribution precisely is called an "ideal gas". The point made here is that perfection need not be a characteristic of crystals only. An IAS structure (Round Cell) follows precisely the specific rules of random packing, and therefore it is perfectly random; any deviations from these rules can be called imperfections (or flaws). A description of possible imperfections in the IAS has been given previously [5]. We discount solids which are crystalline materials but disordered to such an extent that long-range atomic order becomes undetectable. There is an adequate understanding of the observed changes in diffuse X-ray scattering caused by disorder [26].

However, the peak position measured on the experimentally derived scattering pattern (Fig. 7b) is approximately $2.8^{\circ}$ lower that for the imperfection free IAS model (Fig. 7a). This difference cannot be accounted for by the flaws considered here. We have noted that the simplest explanation would be that the atomic radii chosen for the Zr-based IAS model are incorrect, and should be somewhat larger. However, another important factor should be taken into account, namely that vacancies are not stable in an amorphous structure and become randomly distributed throughout the system [27]. The effect of this requires deeper analysis than we are able to present here.

\subsection{Conclusions}

1. In metallic glasses with random atomic arrangements, statistical indeterminacy limits the precise definition of site occupation by atoms; only the probability of nearest-neighbour contacts can be predicted. At a scale larger than a representative volume element, it must be assumed that atomic distribution is homogeneous, in agreement with the overall composition. There cannot be permanency of site identification in amorphous alloys because of the non-existence of a "lattice" and the ease with which mechanisms $\alpha$ and $\beta$ can change cluster composition.

2. Flaws in the random arrangements can be modelled using the ideal amorphous solid, and their effect can be observed by X-ray scattering. However, Round Cells containing more than $10^{6}$ atoms/spheres are required to give a reasonably smooth scattering curve and statistically meaningful results.

3. Ubiquitous $\alpha$ - and $\beta$-mechanisms have been identified and described that are deemed to effect compositional changes in amorphous glassy materials, and may have direct consequences on the mechanical behaviour of amorphous alloys.

4. The ideal amorphous solid model provides the baseline reference for metallic glasses; by definition, it contains no imperfections in its randomness of packing.

5. A clear distinction can be made between truly amorphous solids, as defined by the ideal amorphous solid with no short-, medium- or long-range order, and a disordered solid, in which there is merely no long-range order (e.g. crystalline solids in which the size of the crystals is very small, say, around a nanometre).

6. Analysis of the structure can be carried out by the wellestablished topological measures of Voronoi tessellation and Delauny simplexes.

7. We conclude, with some caution, that a novel science of amorphousness, describing the ideal amorphous atomic arrangements, has been given sufficient basis for further development. The new theory should proceed from the simple, new and powerful unifying idea provided by the concept of ideal amorphous solid.

\section{Acknowledgements}

We are grateful to M.R. James of ANU for the use of ProMac computer for simulation of the IAS structures. Z.H.S. acknowledges helpful correspondence with J.D. FitzGerald of ANU and M. Shimono of NIMS.

\section{References}

[1] Friedrich W, Knipping P, von Laue M. Interferenz-Erscheinungen bei Röntgenstrahlen. Sitzungsberichte der Mathematisch-Physikalischen Classe der Königlich-Bayerischen Akademie der Wissenschaften zu München; 1912:303.

[2] Shechtman D, Blech I, Gratias D, Cahn JW. Phys Rev Lett 1984;53:1951.

[3] Penrose R. Bull Inst Math Appl 1974;10:266.

[4] Sheng HW, Luo WK, Alamgir FM, Bai JM, Ma E. Nature 2006;439:419.

[5] To L-Th, Daley D, Stachurski ZH. Solid State Sci 2006;8:868.

[6] Hui X, Fang HZ, Chen GL, Shang SL, Wang Y, Qin JY, et al. Acta Mater 2009;57:376.

[7] Leopardi P. Electronic transactions on numerical analysis. Kent State University; 2006. 25:309. 
[8] Brostow W, Chybicki M, Laskowski L, Rybicki J. Phys Rev 1998;57:448.

[9] Stachurski ZH. Phys Rev Lett 2003;90/15:5502.

[10] Medvedev NN, Geiger A, Brostow W. J Chem Phys 1990;11:8337.

[11] Clare BW, Kepert DL. Proc Roy Soc Lond Ser A 1986;405:329.

[12] Fürth R. Proc Roy Soc Edinburgh 1964;66:232.

[13] Proffen Th, Neder RD. J Appl Crystall 1997;30:171.

[14] Wang G, Shen J, Sun JF, Zhou BD, Llewellyn DJ, FitzGerald JD, et al. Mater Forum 2005:546.

[15] Guinier A. X-ray diffraction. New York: Freeman \& Co.; 1963.

[16] Makinson JD, Lee JS, Magner SH, Angelis RJ. Adv X Ray Anal 2000;42:407.

[17] Rachek OP. J Non-Cryst Solids 2006;352:3781.

[18] Schuh CA, Hufnagel TC, Ramamurty U. Acta Mater 2007;55:4067.
[19] Frank FC, Kasper JS. Acta Crystall 1958;11:184.

[20] Borodin VA. Philos Mag 1999;79:1887.

[21] Alvarez S. Dalton Trans 2006:2045.

[22] Miracle DB. Nat Mater 2004;3:697.

[23] Martin I, Okhubo T, Ohnuma M, Deconihout B, Hono K. Acta Mater 2004;52:4427.

[24] Liu XJ, Chen GL, Hou HY, Hui X, Yao KF, Lu ZP, et al. Acta Mater 2008;56:2760.

[25] Stachurski ZH, Brostow W. Polimery 2001;46:302.

[26] Welberry TR. Diffuse X-ray scattering and models of disorder. Oxford: Oxford University Press; 2004.

[27] Yavari AR, Le Moulec A, Inoue A, Nishiyama N, Lupu N, Matsubara E, et al. Acta Mater 2005;53:1611. 